Published in final edited form as:

Angiogenesis. 2011 September ; 14(3): 309-319. doi:10.1007/s10456-011-9214-4.

\title{
Rho-Mediated Activation of PI(4)P5K and Lipid Second Messengers is Necessary for Promotion of Angiogenesis by Semaphorin 4D
}

\author{
Nada O. Binmadi ${ }^{1}$, Patrizia Proia ${ }^{1,2}$, Hua Zhou ${ }^{1}$, Yinghua Yang ${ }^{1}$, John R. Basile ${ }^{1,3,4}$ \\ ${ }^{1}$ Department of Oncology and Diagnostic Sciences, University of Maryland Dental School, 650 \\ West Baltimore Street, 7-North, Baltimore, MD 21201, USA \\ 2Department of Sports Science (DISMOT), University of Palermo, Via M. Toselli, 87/B, 90143 \\ Palermo, Italy \\ ${ }^{3}$ Marlene and Stuart Greenebaum Cancer Center, 22 South Greene Street, Baltimore, MD 21201, \\ USA
}

\begin{abstract}
Phosphatidylinositol 4-phosphate 5-kinase ( $\mathrm{PI}(4) \mathrm{P} 5 \mathrm{~K})$ is a type I lipid kinase that generates the lipid second messenger phospholipid phosphatidylinositol 4,5-bisphosphate (PI $(4,5) \mathrm{P} 2)$ and functions downstream of RhoA in actin organization. It is known to play an essential role in neurite remodeling, yielding a phenotype identical to that seen in cells treated with Semaphorin 4D (Sema4D), a protein that regulates proliferation, adhesion and migration in many different cell types. Plexin-B1, the receptor for Sema4D, activates RhoA in order to generate a pro-angiogenic signal in endothelial cells. Therefore, we looked in human umbilical vein endothelial cells (HUVEC) to determine if Plexin-B1 exerted control over the cytoskeleton by regulation of PI(4)P5K activity. Here we demonstrate the Rho/ Rho Kinase (ROK)-dependent generation of $\mathrm{PI}(4,5) \mathrm{P}_{2}$ upon treatment of HUVEC with Sema4D, as well as co-localization of PI(4)P5Ka with Plexin-B1. Formation of $\mathrm{PI}(4,5) \mathrm{P}_{2}$ was necessary for cytoskeletal polymerization, as expression of the phosphatase synaptojanin blocked this effect. We noted phosphorylation and activation of PLC $\gamma$ and an increase in intracellular calcium upon treatment of HUVEC with Sema4D, responses that were necessary for a pro-angiogenic phenotype observed in vitro. Taken together, these results suggest that Plexin-B1 promotes angiogenesis in endothelial cells by signaling through PI(4)P5Ka and generating lipid second messengers.
\end{abstract}

\section{Keywords}

Angiogenesis; Semaphorin 4D; Plexin-B1; Rho; PI(4)P5K; PI(4,5) $\mathrm{P}_{2}$

\footnotetext{
${ }^{4}$ To whom correspondence should be addressed: John R. Basile, Department of Oncology and Diagnostic Sciences, University of Maryland Dental School, 650 West Baltimore Street, 7-North, Baltimore, MD 21201, Tel: 410-706-7937, Fax: 410-706-0519, jbasile@umaryland.edu.
} 


\section{Introduction}

Synthesis of the membrane phospholipid phosphatidylinositol 4,5-bisphosphate $\left(\mathrm{PI}(4,5) \mathrm{P}_{2}\right)$, is an important step in the transduction of intracellular signals. $\mathrm{PI}(4,5) \mathrm{P}_{2}$ is a substrate for phospholipase $\mathrm{C}$ (PLC), serving as a precursor for the formation of the second messengers inositol trisphosphate $\left(\mathrm{IP}_{3}\right)$, which regulates intracellular calcium levels, and diacylglycerol (DAG), a lipid activator of protein kinase $\mathrm{C}(\mathrm{PKC})[1] . \mathrm{PI}(4,5) \mathrm{P}_{2}$ also participates in phosphatidylinositol 3-kinase (PI3K)-mediated pathways, which result in Akt activation and promotion of cell survival and migration. $\mathrm{PI}(4,5) \mathrm{P}_{2}$ can be generated in several different ways but is predominantly synthesized in mammalian cells through the activity of phosphatidylinositol 4-phosphate 5-kinase (PI(4)P5K), a type I lipid kinase that phosphorylates phosphatidylinositol 4-phosphate $(\mathrm{PI}(4) \mathrm{P})$ at the D-5 position of the inositol ring $[2,3]$. Control over the activity of $\mathrm{PI}(4) \mathrm{P} 5 \mathrm{~K}$ is achieved by phosphorylation/ dephosphorylation events and regulation of its subcellular localization. PI(4)P5K shuttles between the nucleus and different regions of the plasma membrane where it is active at focal adhesions promoting actin cytoskeleton polymerization and endocytosis at clathrin coated pits [4].

The small GTPases of the Rho family strongly influence cytoskeletal architecture [5]. Recent studies have suggested that RhoA, acting through its downstream effector Rho Kinase (ROK), exerts its influence, at least in part, through phosphoinositide lipid second messengers [1,4]. It has been observed that RhoA and ROK positively regulate all isoforms of PI(4)P5K, inducing translocation to the membrane where newly formed $\mathrm{PI}(4,5) \mathrm{P}_{2}$ directly interacts with actin-associated proteins to stimulate reorganization of the actin cytoskeleton and trigger stress fiber polymerization, a characteristic of Rho activity $[1,6,7]$.

The semaphorins are a large family of phylogenetically conserved proteins characterized by cysteine-rich semaphorin domains originally identified based on their ability to provide attractive and repulsive axon guidance cues during development [8,9]. There are 27 known semaphorins grouped into eight classes: classes 1 and 2 are invertebrate semaphorins, classes 3 to 7 are found in vertebrates, and an eighth class, class V, has been identified in some nonneurotropic DNA viruses [10]. The main functional receptors for semaphorins are a family of single pass transmembrane proteins known as plexins [11]. There are 9 plexins in vertebrates that have been grouped into 4 families, A through $D$, based on their sequence similarities. When the plexins were first identified they were shown to function in neuronal cell adhesion and nerve bundle fasciculation but have since been identified as regulators of proliferation, adhesion and migration in many different cell types [12]. The nature of the signals generated by semaphorin/ plexin binding is still being deciphered but there is a great deal of evidence that it proceeds through the Rho GTP-ases [13].

The B-family plexins mediate many of the effect of the class 4 semaphorins. We have observed that ligation of Plexin-B1 on endothelial cells by Semaphorin 4D (Sema4D) results in a pro-angiogenic phenotype characterized by endothelial cell migration and tube formation in vitro and enhanced blood vessel growth in vivo [14, 15]. Regulation of Rho activity is necessary for the Plexin-B1-mediated pro-angiogenic phenotype. There is a post synaptic density protein (PSD95), Drosophila disc large tumor suppressor (DlgA), zonula 
occuldens-1 protein (PDZ) binding motif at the C-terminus of Plexin-B1 that associates with PDZ-Rho guanine nucleotide exchange factors (GEF) and leukemia-associated RhoGEF (LARG), proteins that positively regulate Rho [14, 16-19]. Binding of Sema4D to Plexin-B1 results in Rho phosphorylating and activating ROK, which in turn results in the phosphorylation of myosin light chain (MLC) and actomyosin stress fiber formation and contraction [20].

Rho/ ROK signaling through PI(4)P5K leads to $\mathrm{PI}(4,5) \mathrm{P}_{2}$-mediated stress fiber polymerization [7, 21-22], similar to the response we have observed in Sema4D-treated cells [14]. Therefore, we wanted to determine if Plexin-B1 acts through PI(4)P5K as well, and if this step is necessary for the pro-angiogenic phenotype we observe in endothelial cells. Here we show using a system of chimeric receptors, inhibitors, and soluble, purified Sema4D that Plexin-B1 activation results in a RhoA-dependent stimulation of PI(4)P5K, activation of the downstream effector PLC and an increase in intracellular calcium levels. Generation of $\mathrm{PI}(4,5) \mathrm{P}_{2}$ is essential in this process because synaptojanin, an inositol polyphosphate 5-phosphatase that reduces $\mathrm{PI}(4,5) \mathrm{P}_{2}$ levels by hydrolyzing a phosphate group [23], prevents stress fiber polymerization, while endothelial cell migration and tube formation can be blocked by the PLC inhibitor U-73122 and prevention of calcium release or uptake. These results indicate a critical role for RhoA/ ROK-controlled activation of $\mathrm{PI}(4) \mathrm{P} 5 \mathrm{~K}$ and the creation and usage of lipid second messengers in promotion of angiogenesis by Sema4D.

\section{Materials and Methods}

\section{Cell Culture}

Immortalized human umbilical vein endothelial cells (HUVEC) and 293T cells were cultured in DMEM (Sigma, St. Louis, MO) supplemented with $10 \%$ fetal bovine serum and 100 units/ml penicillin/streptomycin/amphotericin B (Sigma).

\section{Trk/Plexin-B1 Fusion Proteins}

Trk-A/Plexin-B1 fusion proteins were made as previously described [18]. Briefly, the intracellular portion of Plexin-B1 with and without its PDZ-binding motif was cut out of the plasmid pCEFL EGFP Plexin-B1 with NheI/NotI and cloned in frame with the extracellular and transmembrane portion of the NGF receptor Trk-A in the vector pCEFL-myc. The constructs were then transfected into cells using Superfect (Qiagen, Valencia, CA).

\section{Production of Soluble Sema4D}

Sema4D was produced and purified as described previously [14]. Briefly, the extracellular portion of Sema4D was subjected to PCR and the resulting product cloned into the plasmid pSecTag2B (Invitrogen, Carlsbad, CA). This construct was transfected into 293T cells growing in serum free media. Media containing soluble Sema4D was collected 65 hours post-transfection and purified with TALON metal affinity resin (Clontech Laboratories, Palo Alto, CA) according to manufacturer's instructions. Concentration and purity of the TALON eluates was determined by SDS PAGE analysis followed by silver staining (Amersham Life Science, Piscataway, NJ) and the Bio-Rad protein assay (Bio-Rad, Hercules, CA). In all 
cases, media collected from cells transfected with the empty pSecTag2B vector were used as control.

\section{Immunoblot Analysis}

Cells were transfected with the indicated constructs, treated with $400 \mathrm{ng} / \mathrm{ml} \mathrm{Sema4D}$ (see above) with or without $25 \mu \mathrm{M}$ fasudil (Calbiochem, San Diego, CA) or transfected with $\mathrm{PI}(4) \mathrm{P} 5 \mathrm{Ka}$ oligos where indicated. Cells were then lysed in lysis buffer $(50 \mathrm{mM}$ Tris- $\mathrm{HCl}$, $150 \mathrm{mM} \mathrm{NaCl}, 1 \% \mathrm{NP} 40)$ supplemented with protease inhibitors $(0.5 \mathrm{mM}$ phenylmethylsulfonyl fluoride, $1 \mu \mathrm{l} / \mathrm{ml}$ aprotinin and leupeptin, Sigma) and phosphatase inhibitors ( $2 \mathrm{mM} \mathrm{NaF}$ and $0.5 \mathrm{mM}$ sodium orthovanadate, Sigma) for 15 minutes at $4^{\circ} \mathrm{C}$. After centrifugation, protein concentrations were measured using the Bio-Rad protein assay (Bio-Rad). $100 \mu \mathrm{g}$ of protein from each sample was subjected to SDS-polyacrylamide gel electrophoresis and transferred onto a PVDF membrane (Immobilon P, Millipore Corp., Billerica, MA). The membranes were then incubated with the appropriate antibodies. The antibodies used were as follows: myc (C-33, Santa Cruz); GAPDH (Sigma); Phospho-PLC $\gamma$ (Cell Signaling Technologies, Danvers, MA); total PLC $\gamma$ (Santa Cruz, Santa Cruz, CA); PI(4)P5Ka (Cell Signaling Technologies); GFP (Cell Signaling Technologies). Proteins were detected using the ECL chemiluminescence system (Pierce, Rockford, IL).

\section{Rho Pull-down Assay}

293T cells expressing TrkA/ full length Plexin-B1 or the $\triangle \mathrm{PDZ}$ mutant chimeric receptor (see above) maintained in DMEM supplemented with $10 \%$ fetal bovine serum were washed in PBS and returned to serum free DMEM for $36 \mathrm{hrs}$. The cells were then washed in PBS and treated with $100 \mathrm{ng} / \mathrm{ml}$ NGF (Upstate, Lake Placid, NY) for the indicated periods of time. Cells were washed with PBS and lysed in lysis buffer (see above). Rho activity was assessed using purified GST-rhotekin-RBD previously bound to Glutathione Sepharose 4B (Amersham Pharmacia, Sweden) to affinity precipitate GTP-bound RhoA. Western blot analysis of total and active Rho was performed using a monoclonal antibody against RhoA (Santa Cruz Biotechnology; 26C4). Immuno-complexes were visualized by enhanced chemiluminescence detection (Amersham Life Science) using goat anti-rabbit coupled to horseradish peroxidase as a secondary antibody (Santa Cruz).

\section{$\mathrm{PI}(4,5) \mathrm{P}_{2}$ Assay}

A PI(4,5) $\mathrm{P}_{2}$ mass ELISA assay (Echelon Biosciences, Salt Lake City, UT) was performed according to manufacturer's instructions. Briefly, 293T cells expressing Trk-A/ PB1 full length or $\triangle \mathrm{PDZ}$ chimeric receptors, with and without $25 \mu \mathrm{M}$ fasudil (Calbiochem) were treated with $100 \mathrm{ng} / \mathrm{ml} \mathrm{NGF}$ for the times indicated, sonicated, and PIP extracted from cell lysates. Samples were incubated with a PIP detector protein, then added to a PIP coated plate for competitive binding. A peroxidase-linked secondary detection reagent and colorimetric substrate was used to detect PIP detector protein binding to the plate, with results determined in a plate reader. PIP $_{2}$ levels are shown relative to untreated cells. 


\section{Immunofluorescence}

293T cells expressing myc tagged Trk-A/PB1 full-length and HA tagged PI(4)P5Ka (the generous gift of Dr. Ralph Isberg) were grown on sterile glass coverslips in 35-mm six-well plates and treated with $100 \mathrm{ng} / \mathrm{ml} \mathrm{NGF}$ for $3 \mathrm{~min}$. The cells were washed in PBS, fixed in $3 \%$ paraformaldehyde for $15 \mathrm{~min}$, and permeabilized in $0.5 \%$ Triton X-100 for $5 \mathrm{~min}$. The cells were then incubated with anti-myc antibody (Santa Cruz) and anti-HA antibody (Covance, Princeton, New Jersey) diluted 1/100 in serum-free Dulbecco's modified Eagle's medium at $4{ }^{\circ} \mathrm{C}$ for $1 \mathrm{~h}$. After three more washings with PBS, coverslips were placed in a humidity chamber for $1 \mathrm{~h}$ and covered with FITC-conjugated anti-rabbit (Sigma, 1:160 dilution in PBS with 3\% fetal bovine serum) and TRITC-conjugated anti-mouse (Jackson Immuno-Research, West Grove, PA, 1:300 dilution in PBS with 3\% fetal bovine serum) secondary antibodies. Coverslips were inverted and mounted onto glass slides with Vectashield containing 4',6-diamidino-2-phenylindole (DAPI, Vector Laboratories, Burlingame, CA) and viewed using a confocal microscope.

\section{Stress Fiber Assay}

HUVEC cells, control transfected or transfected with GFP-tagged synaptojanin (the generous gift of Dr. Pietro DeCamilli) using the electroporation system from Amaxa Biosystems (Gaithersburg, MD) were grown on sterile glass coverslips in 35-mm, 6-well plates and treated for $8 \mathrm{~h}$ with Sema4D, where indicated. Coverslips were placed in a humidity chamber for $1 \mathrm{~h}$ and treated with phalloidin, (AlexaFluor 546-conjugated phalloidin, Invitrogen, Molecular Probes, Eugene, OR) diluted at 1:400 in PBS with 3\% FBS. Coverslips were then inverted and mounted onto glass slides with Vectashield containing DAPI (Vector Laboratories) and viewed with fluorescence microscopy. Images were taken using a SPOT digital camera attached to a Zeiss Axiophot microscope (Carl Zeiss). The number of cells ( $\mathrm{n}>300$ ) exhibiting stress fiber polymerization was counted and the results expressed as a percentage of the total number of cells counted.

\section{$\mathrm{Ca}^{2+}$ Assay}

HUVEC cells were treated with Sema4D for the times indicated and incubated with PBS, $25 \mu \mathrm{M}$ fasudil (Calbiochem) or 10 $\mu \mathrm{M}$ of the PLC inhibitor U-73122 (Sigma). Changes in intracellular $\mathrm{Ca}^{2+}$ levels were measured using Fluo-4 NW calcium indicators (Invitrogen), following the manufacturer's instructions.

\section{Migration Assay}

Media containing 10\% FBS (positive control), and serum free media containing 0.5\% BSA (negative control), $50 \mathrm{ng} / \mathrm{ml}$ of VEGF (R\&D Systems, Minneapolis, MN) or $400 \mathrm{ng} / \mathrm{ml}$ of purified Sema4D along with the indicated inhibitors (500 nM verapamil (Sigma), $25 \mu \mathrm{M}$ BAPTA-AM (Sigma), $25 \mu$ M fasudil (Sigma) and 10 $\mu$ M U-73122 (Sigma)) were placed in the bottom well of a Boyden chamber and served as the chemoattractants. Serum starved HUVEC cells were added to the top chamber. The two chambers were separated by a PVPF membrane (Osmonics, GE Water Technologies, Trevose, PA, $8 \mu \mathrm{m}$ pore size) coated with 10 $\mu \mathrm{g} / \mathrm{ml}$ fibronectin (GIBCO, Carlsbad, CA). The migration assay was then performed as described [24]. Briefly, after $7 \mathrm{~h}$, the chamber was disassembled and the membrane stained 
with Diff-Quick Stain (Diff-Quick, Dade Behring, Deerfield, Illinois), placed on a glass slide and scanned. Densitometric quantitation was performed with NIH image software and cell migration expressed as pixel intensity. Each experiment was performed in triplicate and average and standard deviation calculated.

\section{Tubulogenesis Assay}

HUVEC cells were grown on $35 \mathrm{~mm}$ plates coated with $150 \mu \mathrm{l}$ of Cultrex basement membrane extract (Trevigen, Gaithersburg, MD) and incubated overnight in serum free DMEM with $50 \mathrm{ng} / \mathrm{ml}$ of VEGF or in Sema4D conditioned media, with or without inhibitors (see above). Cells were then fixed in $0.5 \%$ glutaraldehyde and photographed. Media containing $10 \%$ FBS or $0.1 \%$ BSA served as the positive and negative controls, respectively, where indicated. Quantification of results was determined using NIH Image, measuring and summing the length of all tubular structures observed in 10 random fields for three independent experiments.

\section{Results}

\section{Plexin-B1 signaling proceeds through RhoA in a chimeric receptor system}

In order to specifically isolate and study Plexin-B1 signaling, we utilized chimeric receptors consisting of a myc tagged extracellular portion of the rat nerve growth factor receptor TrkA fused to the transmembrane and intracellular segment of human Plexin-B1, with or without the PDZ binding domain necessary for recruitment of the adapter proteins that activate Rho. These receptors have been previously described and used to study Plexin-B1 signaling in a variety of cells $[14,18,24]$. We successfully expressed these receptors in 293T cells (Fig. 1a) and show in a Rho activity assay that when stimulated with NGF, these cells can activate Rho but only when expressing full length intracellular Plexin-B1 containing the intact PDZ binding motif and not the $\triangle$ PDZ mutant (Fig. 1b). These results confirm that full length Plexin-B1 activates Rho and presumably its downstream effectors.

\section{$\mathrm{PI}(4) \mathrm{P} 5 \mathrm{Ka}$ is activated by Plexin-B1 in a Rho-dependent manner}

To investigate if the Rho downstream target PI(4)P5K is activated in Plexin-B1 signaling, we measured PIP(4,5) 2 levels as an indicator of PI(4)P5K activity in 293T cells expressing the wild-type or $\triangle \mathrm{PDZ}$ binding domain mutant receptors, growing in NGF with or without the ROK inhibitor fasudil (Fig. 2a). PIP $(4,5)_{2}$ levels increased in cells expressing the wild-type full length receptor, but not the $\triangle \mathrm{PDZ}$ binding domain mutant or in cells expressing the wild-type receptor but co-treated with fasudil, both of which are incapable of activating Rhomediated pathways (Fig. 2a). To determine if PI(4)P5Ka co-localized with Plexin-B1, tagged forms of the Trk-A/PB1 wt chimeric receptor and PI(4)P5Ka were transfected into 293T cells and immunofluorescence performed in the presence or absence of NGF. While Plexin-B1 localized to the membrane, as expected (Fig. 2b, green), PI(4)P5Ka remained mainly nuclear in untreated cells (Fig. 2b, red). Upon treatment however, the merged image shows that these proteins co-localize (Fig. 2b, right panels). Taken together, these results indicate that activation of Plexin-B1 results in the recruitment of PI(4)P5Ka into a Rho and ROK-dependent signaling complex that generates the lipid second messenger $\operatorname{PIP}(4,5)_{2}$. 


\section{$\operatorname{PIP}(4,5)_{2}$ formation is necessary for Sema4D/ Plexin-B1/ RhoA-mediated polymerization of stress fibers, activation of the PI(4)P5Ka effector PLC and generation of intracellular calcium}

To determine the biological significance of the activation of PI(4)P5Ka and the generation of PIP $(4,5)_{2}$ in Sema4D/ Plexin-B1-mediated angiogenesis, we performed a stress fiber polymerization assay on endothelial cells expressing GFP-tagged synaptojanin, an inositol polyphosphate 5-phosphatase that dephosphorylates $\mathrm{PI}(4,5) \mathrm{P}_{2}\left(\right.$ and $\left.\mathrm{PI}(3,4,5) \mathrm{P}_{3}\right)$ at the $5^{\prime}$ position of the inositol ring [25]. Green cells, and hence those expressing synaptojanin, exhibited a loss of stress fiber polymerization in response to Sema4D compared to neighboring untransfected control cells (Fig. 3a). The results of stress fiber inhibition are shown graphically (Fig. 3a, bottom panel). $\mathrm{PI}(4,5) \mathrm{P}_{2}$ is a substrate for PLC, an important downstream effector of PI(4)P5K that utilizes $\mathrm{PI}(4,5) \mathrm{P}_{2}$ for the generation of the lipid second messengers $\mathrm{IP}_{3}$ and DAG. Therefore, to further investigate the pathways involved, we treated cells with Sema4D with and without the ROK inhibitor fasudil and looked for phosphorylation and activation of PLC $\gamma 1$ in an immunoblot of cell lysates. We observed that cells incubated with Sema4D exhibited phosphorylation and hence activation of PLC $\gamma 1$ but not when incubated with fasudil (Fig. 3b), suggesting that PLC $\gamma 1$ and PI(4)P5K are activated by Sema4D ligation of Plexin-B1 in a RhoA and ROK-dependent manner. Calcium ion release within the cell is a consequence of PLC $\gamma 1$ activity, so we performed an assay to detect calcium levels in Sema4D treated cells growing in fasudil or the PLC $\gamma$ inhibitor U-73122. At approximately $45 \mathrm{~min}$. calcium levels rose dramatically in Sema4D treated cells, but not in those growing in fasudil or U-73122 (Fig. 3c). We have previously demonstrated that activation of Plexin-B1 also leads to phosphorylation of the regulatory subunit of PI3K, another enzyme that utilizes $\mathrm{PI}(4,5) \mathrm{P}_{2}$ as a substrate, but in this case to activate Akt [24]. Taken together, these results indicate a critical role for RhoA/ ROKmediated activation of PI(4)P5Ka and generation of PIP $(4,5)_{2}$ for stress fiber formation, activation of PLC and elevation of intracellular calcium levels.

\section{Activation of PI(4)P5Ka and PLC and generation of intracellular calcium are necessary for the Sema4D/ Plexin-B1-mediated proangiogenic phenotype in endothelial cells}

To determine the biological significance of PLC activation and calcium influx on endothelial cell migration and promotion of angiogenesis, we studied HUVEC cells in a Boyden chamber migration assay with Sema4D as the chemoattractant, with or without verapamil (an inhibitor of extracellular calcium influx), BAPTA-AM (a divalent ion chelator with a high affinity for calcium), the ROK inhibitor fasudil and the PLC inhibitor U-73122. We observed robust HUVEC migration towards Sema4D (and the VEGF positive control) but not towards Sema4D when co-treated with verapamil, BAPTA-AM, fasudil or U-73122 (Fig. 4a). To further explore the pro-angiogenic phenotype and determine the biological significance of $\mathrm{PI}(4) \mathrm{P} 5 \mathrm{Ka}$ activity and $\mathrm{PIP}(4,5)_{2}$ in this process, we looked for tube formation in HUVEC cells growing on reconstituted basement membrane material. We transfected HUVEC with si PI(4)P5Ka oligos and GFP-tagged synaptojanin. Immunoblots show loss of PI(4)P5Ka in oligo transfected cells (Fig. 4b, left panels) and higher levels of synpatojanin in transfeceted HUVEC compared to controls (Fig. 4b, right panels). As expected, we observed formation of capillary tubules in Sema4D treated cells, but not when these cells lost expression of PI(4)P5Ka or were overexpressing synaptojanin (Fig. 4c, 
upper panels). These results were quantified (Fig. 4c, lower panels). Capillary formation was also lost in cells growing in Sema4D and co-treated with verapamil, BAPTA-AM, fasudil or U-73122 (Fig. 4d, quantified in the lower panel). Taken together, these results suggest that the pro-angiogenic phenotype elicited by Sema4D in endothelial cells is dependent upon PI(4)P5Ka, PIP(4,5) $)_{2}$ and RhoA and ROK-mediated activation of PLC and elevation of intracellular calcium levels.

\section{Discussion}

Recent investigations into the signaling mechanisms of the plexins have shown these proteins to be versatile receptors. Similar to the A-family plexins, within the intracellular portion of Plexin-B1 is a split GTPase-activating protein (GAP)-like motif that downregulates R-Ras activity and reduces extracellular matrix-mediated integrin activation when bound by Sema4D, thus affecting cell adhesion and motility. There are also regions that interact with the Rho family GTPases Rnd, Rac and Rho [18, 26-28]. Therefore, it is likely that for most semaphorin/ plexin pairs, binding of the receptor by the respective semaphorin ligand initiates a G-protein signaling cascade, the common downstream target of which is the cytoskeleton. This is consistent with a model where the B-plexins regulate actin and microtubule dynamics upon activation by the semaphorins to exert control over cell motility $[29,30]$.

In addition to the G-proteins, tyrosine kinase activity plays a vital role in plexin signaling. Phosphorylation of MAPK and Akt downstream effectors has been observed following Plexin-B1 activation in some cells [24,31].The plexins themselves are devoid of any intrinsic kinase activity, so this function is provided by kinases recruited to the signaling complex. For example, it has been observed that upon Sema4D ligation Plexin-B1 interacts with c-MET [32], Pyk2 [24], or the epidermal growth factor receptor ERBB2 [33, 34], depending upon the cell type and the context, which then phosphorylate Plexin-B1 in a step required for signaling. Much of the research into the different plexin families indicates that these two features- tyrosine phosphorylation and G-protein signaling and subsequent cytoskeletal alterations- likely govern signaling for most if not all of the plexins [29]. Indeed, our group has observed phosphorylation and activation of Plexin-B1 in Sema4D treated endothelial cells that results in Rho-mediated stress fiber polymerization, cell migration and a pro-angiogenic phenotype [14, 24].

$\mathrm{PI}(4) \mathrm{P} 5 \mathrm{~K}$ isoforms have been shown to function downstream of RhoA and ROK in actin organization $[21,22]$ and play an essential role in neurite remodeling, yielding a phenotype identical to that seen in cells treated with Sema4D [35, 36]. Therefore, we wanted to explore if Plexin-B1 exerted control over the cytoskeleton in endothelial cells at least partially by regulation of $\mathrm{PI}(4) \mathrm{P} 5 \mathrm{Ka}$ activity and $\mathrm{PI}(4,5) \mathrm{P}_{2}$ synthesis. We isolated Plexin-B1 signaling and demonstrated Rho/ ROK-dependent generation of $\mathrm{PI}(4,5) \mathrm{P}_{2}$ upon treatment with Sema4D, as well as translocation of PI(4)P5Ka to the cell membrane and co-localization of these proteins upon Plexin-B1 activation. Formation of PI $(4,5) \mathrm{P}_{2}$ was necessary for cytoskeletal polymerization, a readout for Rho activation and indicator of cell adhesion and potential for mobility, because expression of the phosphatase synaptojanin blocked this effect. Using RNA interference, we demonstrated the importance of PI(4)P5Ka in 
endothelial cell migration and tube formation, as siRNA oligos directed against this enzyme prevented a proangiogenic response in immortalized HUVEC cells.

We also noted that Rho and ROK-mediated activation of PLC $\gamma$ was important in endothelial cell migration since the PLC $\gamma$ inhibitor U-73122 could block this effect. Swiercz et al. have recently shown a novel nonenzymatic function of PLC $\gamma$ linking tyrosine phosphorylation of Plexin-B1 to the regulation of PDZRhoGEF recruitment and downstream RhoA activation (37). In this context, PLC $\gamma$ was biologically significant in mediating in growth cone collapse (37). Similar to their findings, we noted phosphorylation and activation of PLC $\gamma$ upon treatment of HUVECs with Sema4D. However, it appears that in the lipase activity of PLC $\gamma$ was important for the pro-angiogenic phenotype, as we observed an increase in cellular calcium that was necessary for cell migration and tube formation on reconstituted basement membrane material. We don't know yet the mechanism of PLC activation, only that it is dependent upon Rho and ROK. Further investigation is warranted.

Taken together, these results suggest that in addition to altering the cytoskeleton through RRas inhibition and RhoA activation, Plexin-B1 promotes angiogenesis in endothelial cells by signaling through $\mathrm{PI}(4) \mathrm{P} 5 \mathrm{Ka}$ and generating lipid second messengers. With these new findings we propose the model for Plexin-B1 pro-angiogenic signaling in endothelial cells shown in Fig. 5. Upon binding Sema4D, Plexin-B1 recruits PDZ-RhoGEF and LARG in addition to acting as a Ras GAP and activating a MAP kinase cascade. Rho activation proceeds through ROK to activate $\mathrm{PI}(4) \mathrm{P} 5 \mathrm{Ka}$, creating a pool of $\mathrm{PI}(4,5) \mathrm{P}_{2}$. Rho and ROKdependent phosphorylation and activation of $\mathrm{PLC} \gamma$ and $\mathrm{PI}(3) \mathrm{K}$ follows (Fig. 5). PLC $\gamma$ in turn generates $\mathrm{IP}_{3}$ which causes an increase in intracellular calcium, a phenomenon we observed in Sema4D treated endothelial cells that was necessary for cell migration and capillary tube formation in vitro. An accumulation of calcium and activation of the downstream effectors such as PKC and Akt leads to actin fiber polymerization, contraction and cell movement. Further analysis of downstream targets, their effectors, and the mechanisms of actin polymerization remains to be studied.

\section{Acknowledgemets}

We would like to thank Dr. Ralph Isberg for the generous gift of the PI(4)P5Ka plasmid and Dr. Pietro DeCamilli for the synaptojanin construct. Dr. Nada Binmadi gratefully acknowledges King Abdulaziz University in Jeddah, Saudi Arabia for a doctorate scholarship.

\section{References}

1. Weernink PA, Meletiadis K, Hommeltenberg S, Hinz M, Ishihar, H, Schmidt M, Jakobs KH (2004) Activation of type I phosphatidylinositol 4-phosphate 5-kinase isoforms by the Rho GTPases, RhoA, Rac1, and Cdc42. J Biol Chem 279(9):7840-7849 [PubMed: 14681219]

2. Rameh LE, Tolias KF, Duckworth BC, Cantley LC (1997) A new pathway for synthesis of phosphatidylinositol-4,5-bisphosphate. Nature 390(6656):192-196 [PubMed: 9367159]

3. Zhang X, Loijens JC, Boronenkov IV, Parker GJ, Norris FA, Chen J, Thum O, Prestwich GD, Majerus PW, Anderson RA (1997) Phosphatidylinositol-4-phosphate 5-kinase isozymes catalyze the synthesis of 3-phosphate-containing phosphatidylinositol signaling molecules. J Biol Chem 272(28):17756-17761 [PubMed: 9211928]

4. Oude Weernink PA, Schmidt M, Jakobs KH (2004) Regulation and cellular roles of phosphoinositide 5-kinases. Eur J Pharmacol 500(1-3):87-99 [PubMed: 15464023] 
5. Etienne-Manneville S, Hall A (2002) Rho GTPases in cell biology. Nature 420(6916):629-635 [PubMed: 12478284]

6. Chatah NE, Abrams CS (2001) G-protein-coupled receptor activation induces the membrane translocation and activation of phosphatidylinositol-4-phosphate 5-kinase I alpha by a Rac- and Rho-dependent pathway. J Biol Chem 276(36):34059-34065 [PubMed: 11431481]

7. Toker A (1998) The synthesis and cellular roles of phosphatidylinositol 4,5-bisphosphate. Curr Opin Cell Biol 10(2):254-261 [PubMed: 9561850]

8. Kolodkin AL, Matthes DJ, O'Connor TP, Patel NH, Admon A, Bentley D, Goodman CS (1992) Fasciclin IV: sequence, expression, and function during growth cone guidance in the grasshopper embryo. Neuron 9(5):831-845 [PubMed: 1418998]

9. Kolodkin AL, Matthes DJ, Goodman CS (1993) The semaphorin genes encode a family of transmembrane and secreted growth cone guidance molecules. Cell 75(7):1389-1399 [PubMed: 8269517]

10. Yazdani U, Terman JR (2006) The semaphorins. Genome Biol 7(3):211 [PubMed: 16584533]

11. Tamagnone L, Artigiani S, Chen H, He Z, Ming GI, Song H, Chedotal A, Winberg ML, Goodman CS, Poo M, Tessier-Lavigne M, Comoglio PM (1999) Plexins are a large family of receptors for transmembrane, secreted, and GPI-anchored semaphorins in vertebrates. Cell 99(1):71-80 [PubMed: 10520995]

12. Tamagnone L, Comoglio PM (2000) Signalling by semaphorin receptors: cell guidance and beyond. Trends Cell Biol 10(9):377-383 [PubMed: 10932095]

13. Negishi M, Oinuma I, Katoh H (2005) Plexins: axon guidance and signal transduction. Cell \& Mol Life Sci 62(12):1363-1371 [PubMed: 15818466]

14. Basile JR, Barac A, Zhu T, Guan KL, Gutkind JS (2004) Class IV semaphorins promote angiogenesis by stimulating Rho-initiated pathways through plexin-B. Cancer Res 64(15):52125224 [PubMed: 15289326]

15. Basile JR, Castilho RM, Williams VP, Gutkind JS (2006) Semaphorin 4D provides a link between axon guidance processes and tumor-induced angiogenesis. PNAS 103(24):9017-9022 [PubMed: 16754882]

16. Aurandt J, Vikis HG, Gutkind JS, Ahn N, Guan KL (2002) The semaphorin receptor plexin-B1 signals through a direct interaction with the Rho-specific nucleotide exchange factor, LARG. Proc Natl Acad Sci U S A 99(19):12085-12090 [PubMed: 12196628]

17. Hirotani M, Ohoka Y, Yamamoto T, Nirasawa H, Furuyama T, Kogo M, Matsuya T, Inagaki S (2002) Interaction of plexin-B1 with PDZ domain-containing Rho guanine nucleotide exchange factors. Biochem Biophys Res Commun 297(1):32-37 [PubMed: 12220504]

18. Perrot V, Vazquez-Prado J, Gutkind JS (2002) Plexin B regulates Rho through the guanine nucleotide exchange factors leukemia-associated Rho GEF (LARG) and PDZ-RhoGEF. J Biol Chem 277(45):43115-43120 [PubMed: 12183458]

19. Swiercz JM, Kuner R, Behrens J, Offermanns S (2002) Plexin-B1 directly interacts with PDZRhoGEF/LARG to regulate RhoA and growth cone morphology. Neuron 35(1):51-63 [PubMed: 12123608]

20. Basile JR, Gavard J, Gutkind JS (2007) Plexin-B1 utilizes RhoA and Rho kinase to promote the integrin-dependent activation of Akt and ERK and endothelial cell motility. J Biol Chem 282(48):34888-34895 [PubMed: 17855350]

21. Chong LD, Traynor-Kaplan A, Bokoch GM, Schwartz MA (1994) The small GTP-binding protein Rho regulates a phosphatidylinositol 4-phosphate 5-kinase in mammalian cells. Cell 79(3):507513 [PubMed: 7954816]

22. Shibasaki Y, Ishihara H, Kizuki N, Asano T, Oka Y, Yazaki Y (1997) Massive actin polymerization induced by phosphatidylinositol-4-phosphate 5-kinase in vivo. J Biol Chem 272(12):7578-7581 [PubMed: 9065410]

23. Mitchell CA, Brown S, Campbell JK, Munday AD, Speed CJ (1996) Regulation of second messengers by the inositol polyphosphate 5-phosphatases. Biochem Soc Trans 24(4):994-1000 [PubMed: 8968499] 
24. Basile JR, Afkhami T, Gutkind JS (2005) Semaphorin 4D/plexin-B1 induces endothelial cell migration through the activation of PYK2, Src, and the phosphatidylinositol 3-kinase-Akt pathway. Mol Cell Biol 25(16):6889-6898 [PubMed: 16055703]

25. Perera RM, Zoncu R, Lucast L, De Camilli P, Toomre D (2006) Two synaptojanin 1 isoforms are recruited to clathrin-coated pits at different stages. Proc Natl Acad Sci U S A 103(51):1933219337 [PubMed: 17158794]

26. Oinuma I, Katoh H, Negishi M (2006) Semaphorin 4D/Plexin-B1-mediated R-Ras GAP activity inhibits cell migration by regulating \{beta\} 1 integrin activity. J Cell Biol 173(4):601-13 [PubMed: 16702230]

27. Oinuma I, Ishikawa Y, Katoh H, Negishi M (2004) The Semaphorin 4D receptor Plexin-B1 is a GTPase activating protein for R-Ras. Science 305(5685):862-865 [PubMed: 15297673]

28. Oinuma I, Katoh H, Negishi M (2004) Molecular dissection of the semaphorin 4D receptor plexinB1-stimulated R-Ras GTPase-activating protein activity and neurite remodeling in hippocampal neurons. J Neurosci 24(50):11473-11480 [PubMed: 15601954]

29. Tamagnone L, Comoglio PM (2004) To move or not to move? Semaphorin signalling in cell migration. EMBO Rep 5(4):356-361 [PubMed: 15060572]

30. Kruger RP, Aurandt J, Guan KL (2005) Semaphorins command cells to move. Nature Rev Mol Cell Biol 6(10):789-800 [PubMed: 16314868]

31. Aurandt J, Li W, Guan KL (2006) Semaphorin 4D activates the MAPK pathway downstream of plexin-B1. Biochem J 394(Pt 2):459-464 [PubMed: 16187944]

32. Giordano S, Corso S, Conrotto P, Artigiani S, Gilestro G, Barberis D, Tamagnone L, Comoglio PM (2002) The semaphorin 4D receptor controls invasive growth by coupling with Met. Nat Cell Biol 4(9):720-724 [PubMed: 12198496]

33. Swiercz JM, Kuner R, Offermanns S (2004) Plexin-B1/RhoGEF-mediated RhoA activation involves the receptor tyrosine kinase ErbB-2. J Cell Biol 165(6):869-880 [PubMed: 15210733]

34. Swiercz JM, Worzfeld T, Offermanns S (2008) ErbB-2 and met reciprocally regulate cellular signaling via plexin-B1. J Biol Chem 283(4):1893-1901 [PubMed: 18025083]

35. van Horck FP, Lavazais E, Eickholt BJ, Moolenaar WH, Divecha N (2002) Essential role of type I(alpha) phosphatidylinositol 4-phosphate 5-kinase in neurite remodeling. Curr Biol 12(3):241245 [PubMed: 11839279]

36. Yamazaki M, Miyazaki H, Watanabe H, Sasaki T, Maehama T, Frohman MA, Kanaho (2002) Phosphatidylinositol 4-phosphate 5-kinase is essential for ROCK-mediated neurite remodeling. J Biol Chem 277(19):17226-17230 [PubMed: 11877391]

37. Swiercz JM, Worzfeld T, Offermanns S (2009) Semaphorin 4D signaling requires the recruitment of phospholipase C gamma into the plexin-B1 receptor complex. Mol Cell Biol 29(23):6321-6334 [PubMed: 19805522] 

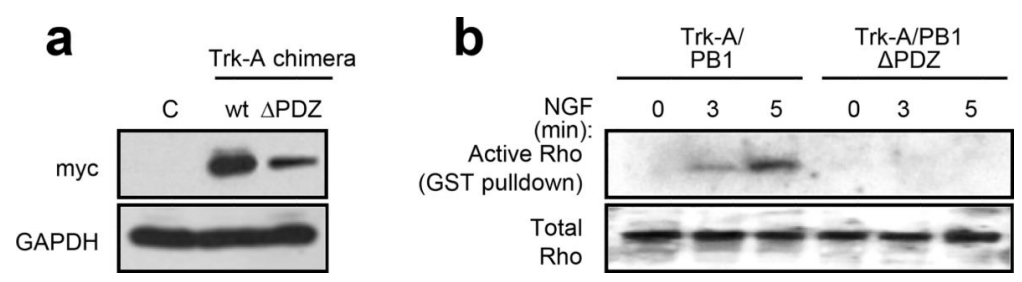

Fig. 1.

Plexin-B1 activates RhoA. (a) Trk-A/ full length Plexin-B1 (wt) and a chimeric construct lacking the PDZ binding motif ( $\triangle \mathrm{PDZ}$ ) were transfected into 293T cells. Expression was confirmed with an immunoblot for the myc tag (top panel). GAPDH was used as a loading control (lower panel). (b) $293 \mathrm{~T}$ cells expressing the chimeric receptors were treated with 100ng/ml NGF for the indicated times and subjected to a pulldown assay, looking for active, GTP-bound RhoA. Only the full length receptor (Trk-A/PB1) could bind active Rho in the pulldown, and not the truncated mutant (Trk-A/PB1 $\triangle \mathrm{PDZ}$ ) lacking the PDZ binding motif. 
a
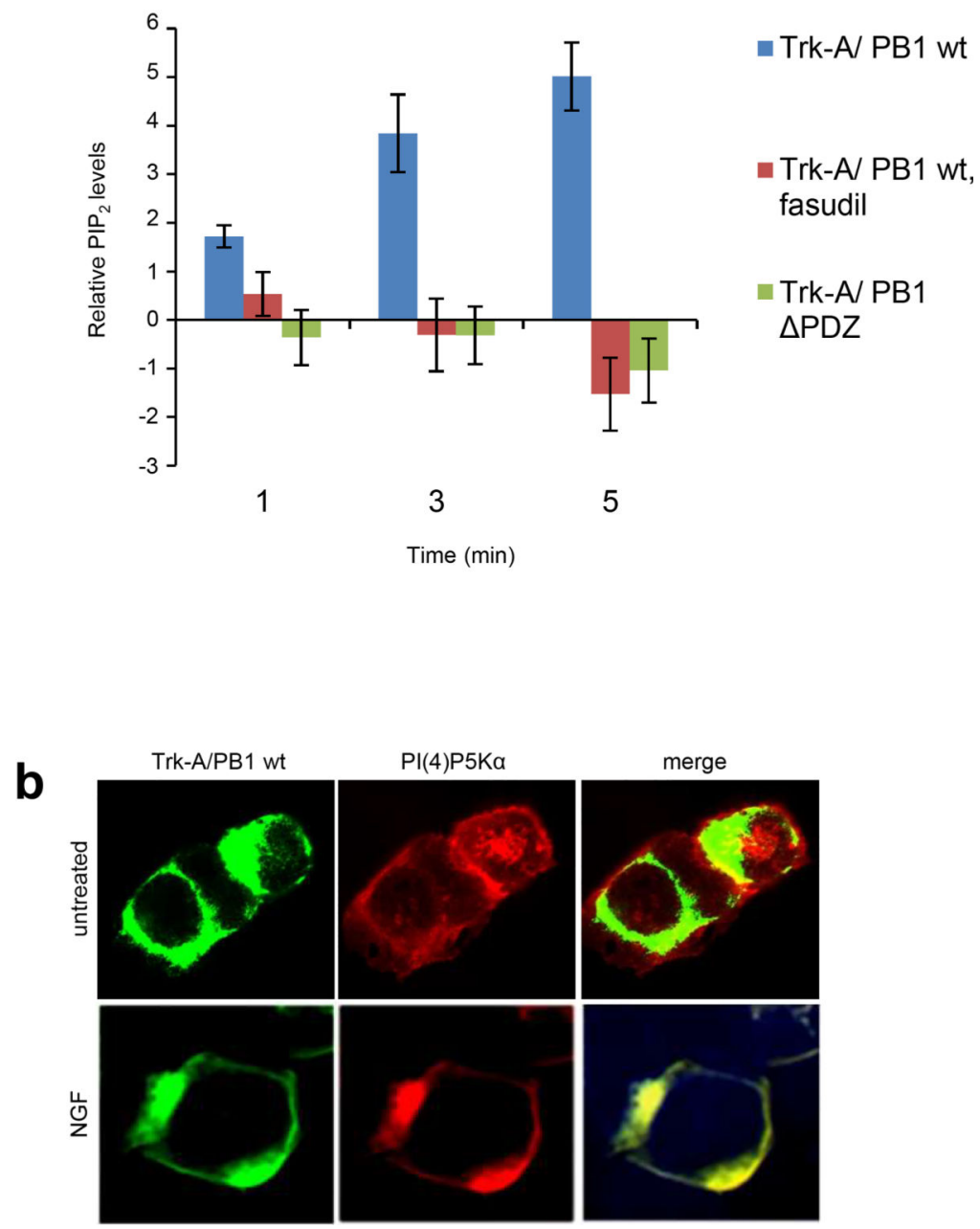

Fig. 2.

$\mathrm{PI}(4) \mathrm{P} 5 \mathrm{Ka}$ is activated by Sema4D/ Plexin-B1 in a Rho-dependent manner and co-localizes with Plexin-B1. (a) A PIP 2 assay was performed on 293 T cells expressing the Trk-A/ full length wild-type Plexin-B1 chimera (Trk-A/PB1 wt, blue bars), Trk-A/ full length Plexin-B1 in the presence of fasudil (Trk-A/PB1 wt, fasudil, dark red bars) and the construct lacking the PDZ binding motif (Trk-A/PB1 $\triangle$ PDZ, green bars), treated with 100ng/ml NGF for the times indicated. Levels of $\mathrm{PIP}_{2}$ relative to untreated cells are shown on the Y-axis. (b) Immunofluorescence for tagged forms of Trk-A/PB1 wt (green, left panel) and PI(4)P5Ka (red, center panel) was performed on transfected 293T cells, in the presence or absence of NGF. The merged images (right panels) show migration of PI(4)P5Ka to the membrane upon activation of Sema4D and co-localization of these proteins. 
a
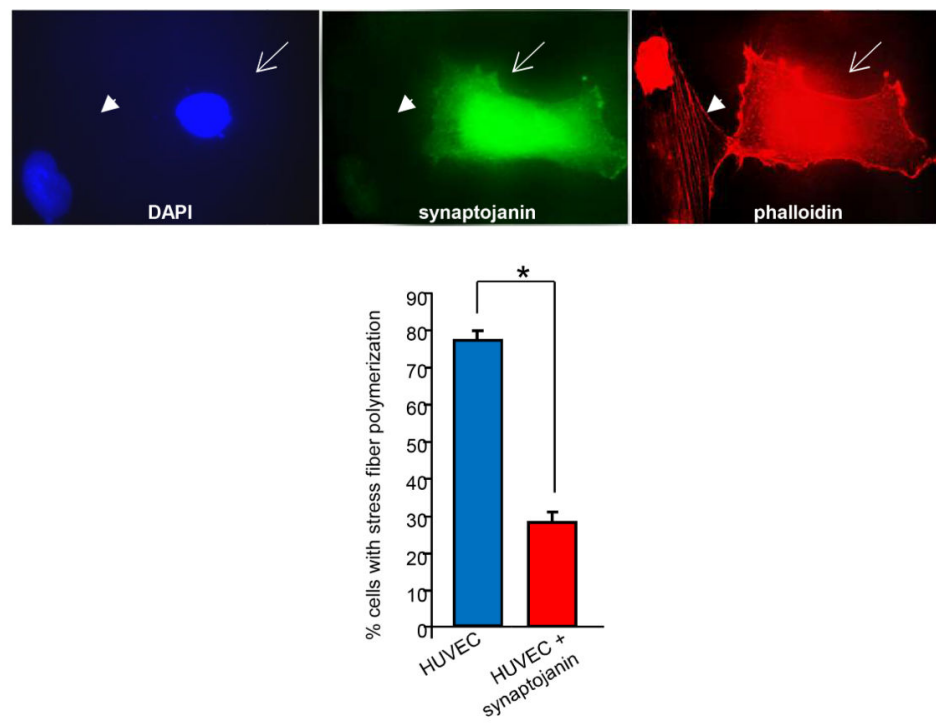

b

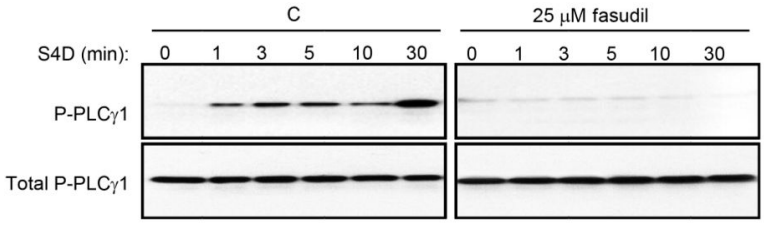

C

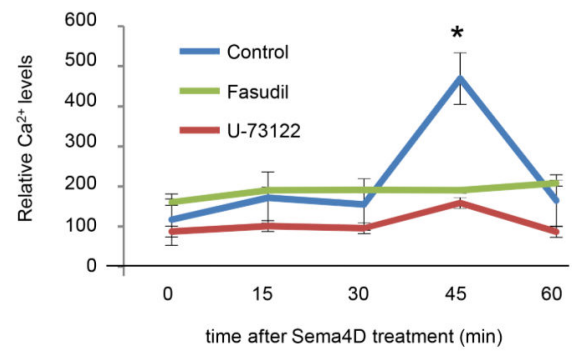

Fig. 3.

$\mathrm{PIP}(4,5)_{2}$ formation is necessary for Sema4D/ Plexin-B1/ RhoA-mediated polymerization of stress fibers, activation of PLC and generation of intracellular calcium. (a) HUVEC expressing GFP-tagged synaptojanin (middle panel) were treated with Sema4D and analyzed using phalloidin staining for stress fiber formation (right panel). Cells expressing synaptojanin (white arrow), exhibited a loss of stress fiber polymerization compared to neighboring untransfected control cells (white arrowhead). DAPI was used as a nuclear stain (left panel). The results of stress fiber inhibition are shown graphically in the bottom panel (*, p< 0.05). (b) An immunoblot of HUVEC treated with Sema4D with or without the ROK inhibitor fasudil for the phosphorylated form of PLC $\gamma 1$ (upper panel). Total PLC $\gamma 1$ was used as a loading control (lower panel). (c) A calcium assay was performed on HUVEC treated with Sema4D and fasudil or the PLC $\gamma$ inhibitor U-73122, measured at the times indicated. Intracellular calcium levels rose dramatically in Sema4D treated cells, but not in those growing in fasudil or U-73122 (*, p<0.05). 


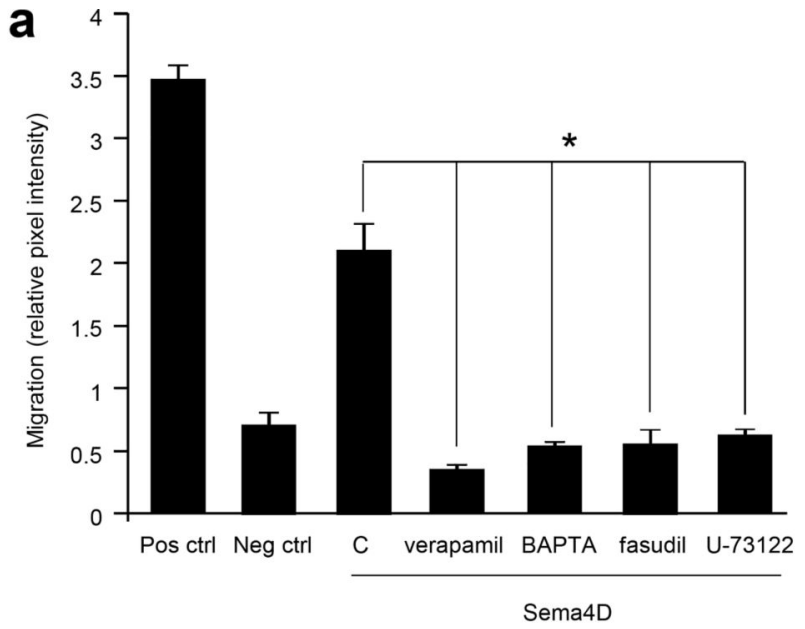

b

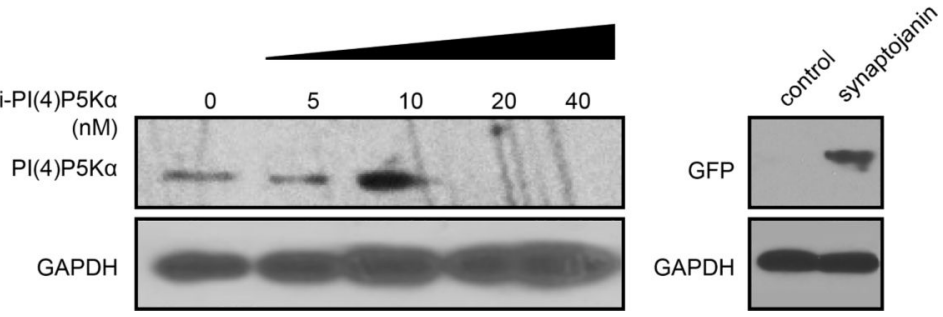


C
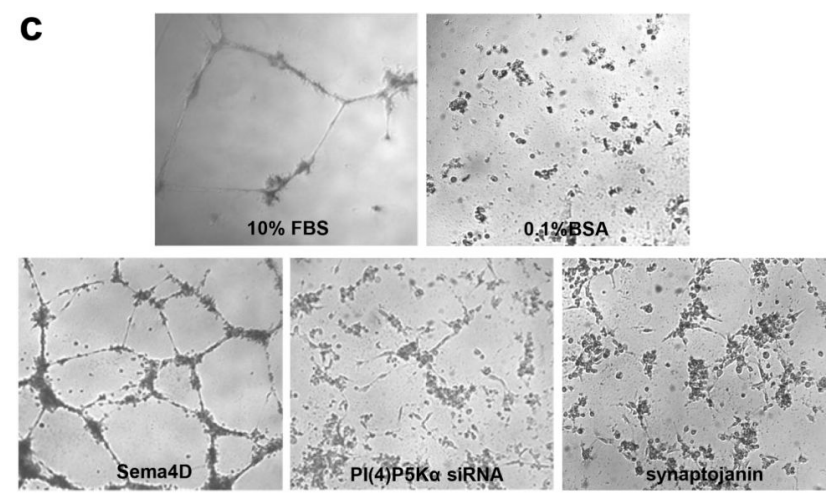

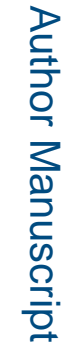

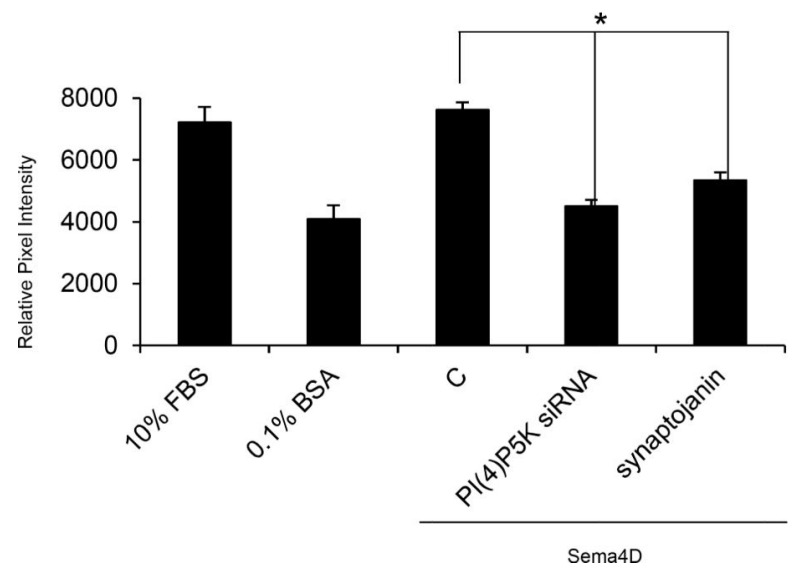

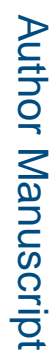

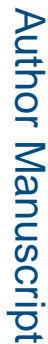

Angiogenesis. Author manuscript; available in PMC 2020 June 29. 

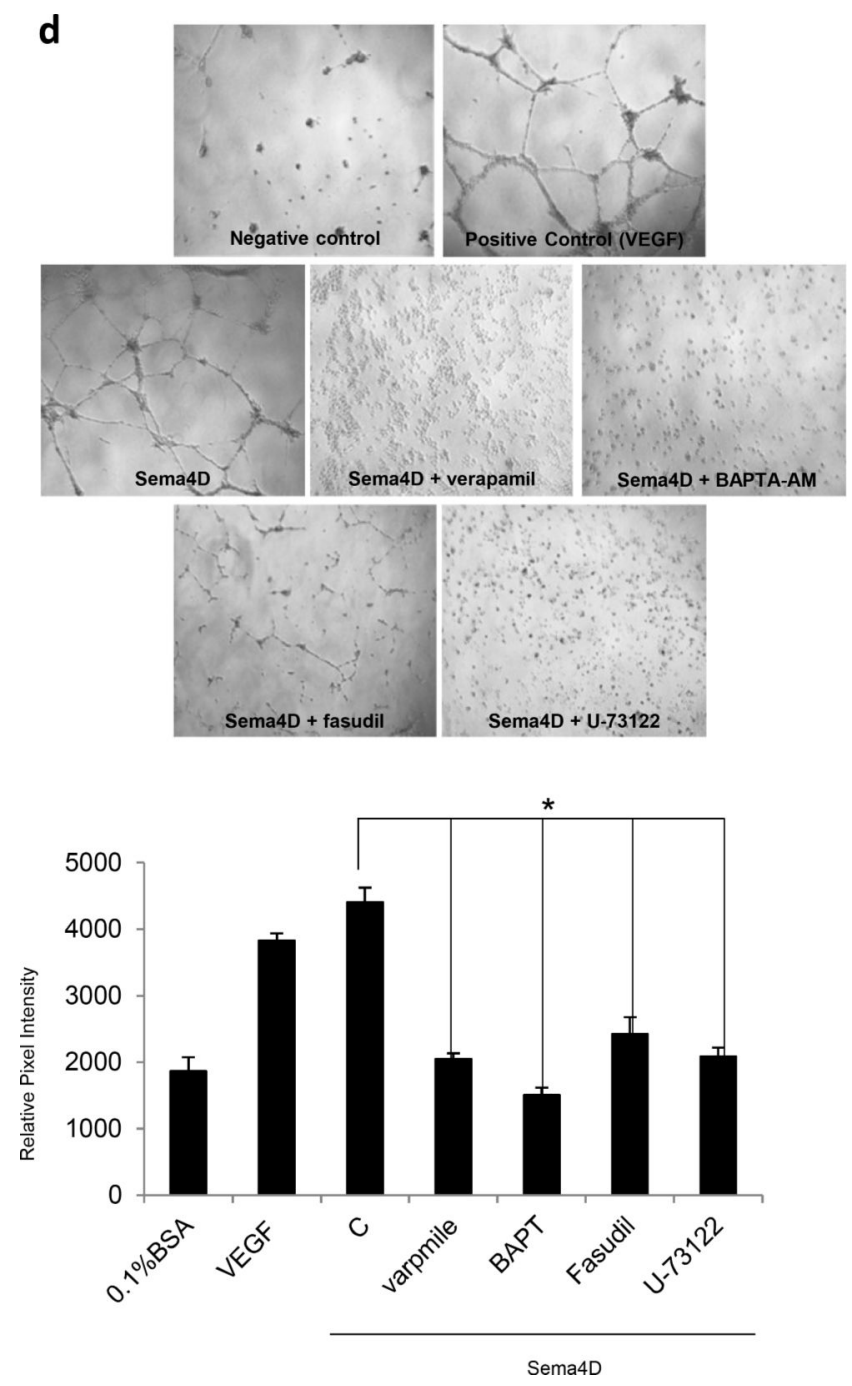

Fig. 4.

Calcium generation, PLC activation and PI(4)P5Ka activity are necessary for the Sema4D/ Plexin-B1-mediated proangiogenic phenotype in endothelial cells. (a) A Boyden chamber migration assay was performed on HUVEC cells with Sema4D as the chemoattractant, with or without verapamil, BAPTA-AM, the ROK inhibitor fasudil and the PLC inhibitor U-73122. Migration (pixel intensity of stained cells, Y-axis) was inhibited towards Sema4D when co-treated with verapamil, BAPTA-AM or U-73122. Positive control was migration towards $10 \%$ FBS. Negative control was migration towards $0.1 \%$ BSA (*, p< 0.05$)$. (b) HUVEC were transfected with si oligos for PI(4)P5Ka (left panels) or with synaptojanin (right panels) and protein levels measured in an immunoblot. (c) HUVEC transfected with si oligos for PI(4)P5Ka or with synaptojanin were grown on reconstituted basement membrane material and evaluated for tube formation in the presence of Sema4D (upper panel). Tube formation is decreased in cells no longer expressing PI(4)P5Ka or over expressing synaptojanin (positive control is HUVECs growing in 10\% FBS, negative control in $1 \%$ BSA). The results are quantified in the graph (lower panel, *, p< 0.05). (d) HUVEC were grown on reconstituted basement membrane material (upper panels) and evaluated for 
tube formation in the presence of Sema4D with or without verapamil, BAPTA-AM, fasudil and the PLC inhibitor U-73122 (positive control is VEGF and negative control 1\% BSA). The results are quantified in the graph (lower panel, *, $\mathrm{p}<0.05$ ). 


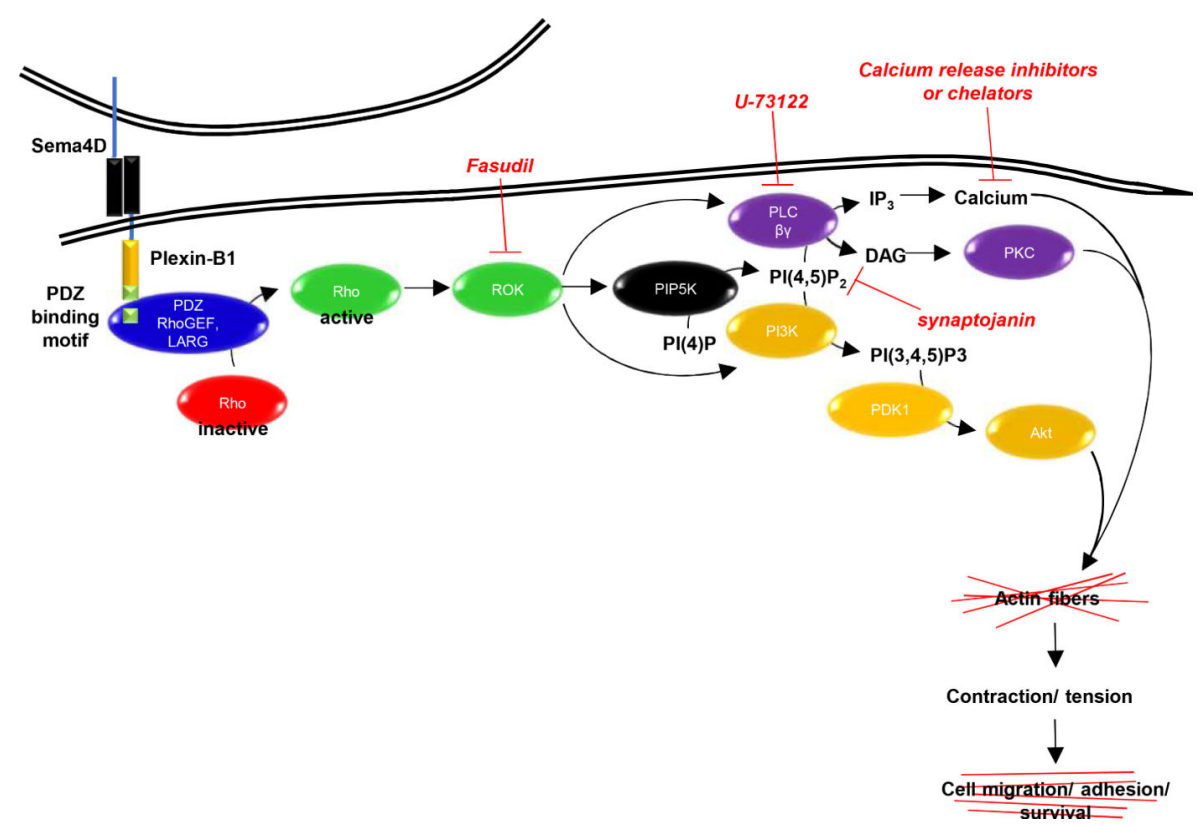

Fig. 5:

A model for Sema4D/ Plexin-B1-mediated proangiogenic signaling in endothelial cells. Upon ligation with Sema4D, Plexin-B1 recruits PDZ RhoGEF and LARG, activating RhoA and the downstream Rho effector ROK. ROK in turn activates PI(4)P5Ka which generates the PIP $(4,5)_{2}$ that serves as a substrate for PI3K and PLC, which also are activated in a RhoA/ ROK-dependent manner. Akt and PLC activation and the subsequent increase in intracellular calcium are necessary for stress fiber polymerization and contraction and the pro-angiogenic, motile phenotype observed in Sema4D treated endothelial cells. 